Proceedings of the 43rd "Jaszowiec", International School and Conference on the Physics of Semiconductors, Wisła 2014

\title{
Type I CdSe and CdMgSe Quantum Wells
}

\author{
R. Rudniewski*, J.-G. Rousset, E. Janik, P. Kossacki, A. Golnik, \\ M. NAWROCKI AND W. PACUSKI
}

Institute of Experimental Physics, Faculty of Physics, University of Warsaw, Hoża 69, 02-681 Warszawa, Poland

In this work we present the band gap engineering, epitaxial growth and optical characterization of $\mathrm{CdSe} / \mathrm{Cd}_{0.9} \mathrm{Mgg}_{0.1}$ Se and $\mathrm{Cd}_{0.9} \mathrm{Mg}_{0.1} \mathrm{Se} / \mathrm{Cd}_{0.85} \mathrm{Mg}_{0.15}$ Se quantum wells with a thickness ranging from 1 to $15 \mathrm{~nm}$. These structures exhibit strong near-band-gap photoluminescence from helium up to room temperature. The emission energy is tuned in the range from 1.74 to $2.1 \mathrm{eV}$ at $7 \mathrm{~K}$, depending on the thickness and well composition. The most intense photoluminescence (both at 7 and $300 \mathrm{~K}$ ) was observed for $10 \mathrm{~nm}$ thick $\mathrm{CdSe} / \mathrm{Cd}_{0.9} \mathrm{Mg}_{0.1} \mathrm{Se}$ wells. Such a structure gives also a sharp emission line $(\mathrm{FWHM}=20 \mathrm{meV})$ at low temperature. The presented quantum wells are well suited for being embedded in lattice matched ZnTe based microcavities.

DOI: 10.12693/APhysPolA.126.1167

PACS: 78.20.-e, 81.05.Dz, 81.07.-b

\section{Introduction}

Recent development of ZnTe-based distributed Bragg reflectors [1] and ZnTe-based microcavities [2-7] opens new perspective for the growth of ZnTe based microcavities with almost lattice matched CdSe quantum wells (QWs). ZnTe based microcavities were initially designed for CdTe quantum dots (QDs), however there are several reasons for the replacement of CdTe QDs by CdSe QWs. First, the photoluminescence (PL) intensity of selenide nanostructures is in general higher. This difference is most striking at room temperature. Second, using wells with very high oscillator strength gives hope for the observation of microcavity polaritons [8], including room temperature observation [9]. Finally, the energy gap of $(\mathrm{Cd}, \mathrm{Mg}) \mathrm{Se}$ can be tuned in a wide range, from red to $\mathrm{UV}$, by varying the $\mathrm{Mg}$ content. This gives hope for applications in the yellow range optoelectronics [10], where other material systems are not very effective. Motivated

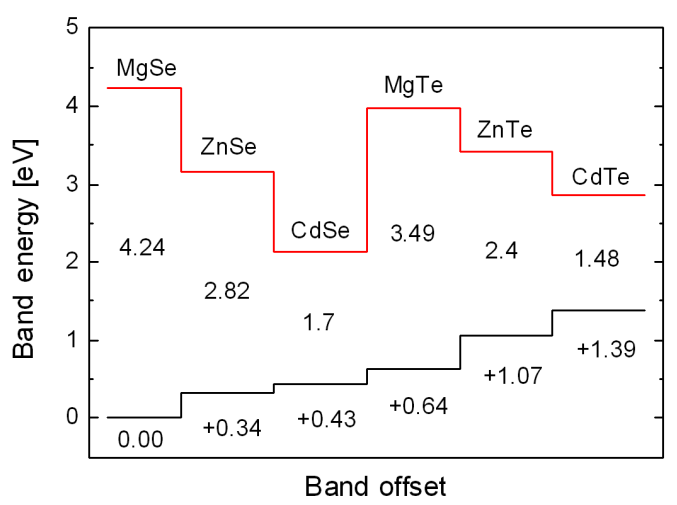

Fig. 1. Valence band offsets of materials used. Data based on the literature data [18-21]. In CdSe/ZnTe heterostructures, the potential is a trap for the electrons but a barrier for holes. By using replacing $\mathrm{ZnTe}$ by $\mathrm{CdMgSe}$, the potential is a trap both for electrons and holes.

${ }^{*}$ corresponding author; e-mail: rr294206@okwf .fuw.edu.pl

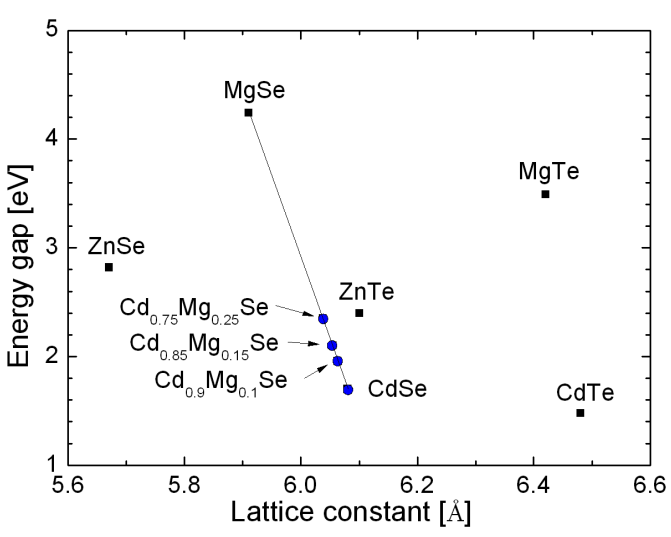

Fig. 2. Band gap and lattice constant of various II-VI semiconductors. The blue dots figure the QW and barrier materials reported in the present work.

by the above reasons we decided to develop CdSe based quantum wells, which might be grown inside ZnTe based microcavities.

The main challenge was to design an appropriate barrier material (Fig. 1), which is almost lattice matched to ZnTe and which assures type I CdSe QW. So it has to exhibit a binding potential for both holes and electrons when combined with CdSe. ZnTe is not a good candidate, because it results in type II CdSe/ZnTe QW [11, 12]. ZnSe exhibits an appropriate electronic structure, but must be excluded due to the large lattice constants mismatch (Fig. 2). MgSe is also inapplicable for the growth of microcavity in the zinc blende structure. Indeed, MgSe barriers have tendency to relax and switch the crystal structure to rock salt or wurtzite structure $[13,14]$. In addition, the hygroscopic character of $\mathrm{Mg}$ rich (more than $60 \%$ ) layers make them rapidly degrade $[15,16]$. Then we decided to use (Cd,Mg)Se as a barrier material with a sufficiently low $\mathrm{Mg}$ content to preserve the pseudomorphic growth regime on $\mathrm{ZnTe}$ [17]. According to Fig. 1 and Refs. [18, 19], (Cd,Mg)Se assures a barrier for both holes and electrons in CdSe QWs. Type I alignment is also confirmed by high PL intensity, which is orders of magnitude stronger in case of $\mathrm{CdSe} / \mathrm{CdMgSe}$ structures than in CdSe/ZnTe (type II) QW. 


\section{Growth}

We used molecular beam epitaxy in order to grow $\mathrm{CdSe} / \mathrm{Cd}_{0.9} \mathrm{Mg}_{0.1}$ Se and $\mathrm{Cd}_{0.9} \mathrm{Mg}_{0.1} \mathrm{Se} / \mathrm{Cd}_{0.85} \mathrm{Mg}_{0.15} \mathrm{Se}$ quantum wells (Fig. 2) with thickness from 1 to $15 \mathrm{~nm}$. The selenide based structures were deposited on a 2-3 $\mu \mathrm{m}$ thick ZnTe buffer grown on GaAs (100) substrates at the temperature of $335{ }^{\circ} \mathrm{C}$. The ZnTe buffers were prepared in a separate growth process. Only a thin ZnTe layer was deposited just before starting the $\mathrm{CdSe}$ or $(\mathrm{Cd}, \mathrm{Mg}) \mathrm{Se}$ growth.

An in situ white light reflectivity setup allowed us to determine the layer thickness and growth rate through the observation of the interference evolution in the thin film. The growth rate of CdSe was about $400 \mathrm{~nm} / \mathrm{h}$. Reflection high-energy electron diffraction (RHEED) was used to evaluate the smoothness of the epitaxial layers. Interestingly, we do not observe any evolution of the RHEED pattern when we switch from ZnTe to CdSe or $\mathrm{Cd}_{0.85} \mathrm{Mg}_{0.15} \mathrm{Se}$. This shows that the growth in zinc blende structure for (100) direction is preserved. The magnesium concentration in $\mathrm{Cd}_{0.85} \mathrm{Mg}_{0.15}$ Se was determined by measuring the band gap energy of $(\mathrm{Cd}, \mathrm{Mg}) \mathrm{Se}$ layers (2.1 eV at helium temperature), which was compared to the data given in Ref. [22]. At the temperature of $40 \mathrm{~K}$ they obtain $E_{\mathrm{g}}=1.82+1.84 x$, where $x$ refers to the magnesium concentration. During the growth of the samples consisting of layers with different magnesium concentrations $\left(\mathrm{Cd}_{0.9} \mathrm{Mg}_{0.1} \mathrm{Se} / \mathrm{Cd}_{0.85} \mathrm{Mg}_{0.15} \mathrm{Se}\right)$ we used two effusion cells with an adequate calibration of molecular beam equivalent pressure.

\section{Optical spectroscopy}

Depending on the application, our wells are intended to be used at helium or room temperature. Therefore our photoluminescence studies were conducted at the temperatures of $7 \mathrm{~K}$ and $300 \mathrm{~K}$. For the excitation we used a continuous wave $405 \mathrm{~nm}$ laser diode. We observed a high impact of the QW width on the PL spectra.

At helium temperatures, the increase of the $\mathrm{CdSe} / \mathrm{Cd}_{0.9} \mathrm{Mg}_{0.1}$ Se QWs width from $1 \mathrm{~nm}$ to $15 \mathrm{~nm}$ results in an energy shift of the emission from 1.74 to $1.86 \mathrm{eV}$. The most intense photoluminescence spectra are exhibited by $10 \mathrm{~nm}$ and $5 \mathrm{~nm}$ thick QW (Fig. 3). For these structures, at low temperatures, the emission line is intense and sharp which allows using these QWs as the optically active material in microcavities. The smallest linewidth, $20 \mathrm{meV}$, was observed for the $5 \mathrm{~nm}$ thick QW. Defect luminescence, which is very pronounced for thick CdSe layers, was almost not visible for our QW structures. Asymmetric shapes of obtained emission lines could be explained by thickness fluctuation of the wells relative to the barrier because of similar material composition. It also causes broadening of linewidth for narrow structure. In case of structure consisting of $1 \mathrm{~nm}$ QW we observe merge of photoluminescence of the QW with barrier.
At room temperature photoluminescence of the QW exhibited significant increase of PL linewidth and an energy shift of the emission from 1.81 to 1.69 to eV. Also in this case the $10 \mathrm{~nm}$ and $5 \mathrm{~nm}$ thick QWs exhibit the most intense photoluminescence (Fig. 4).

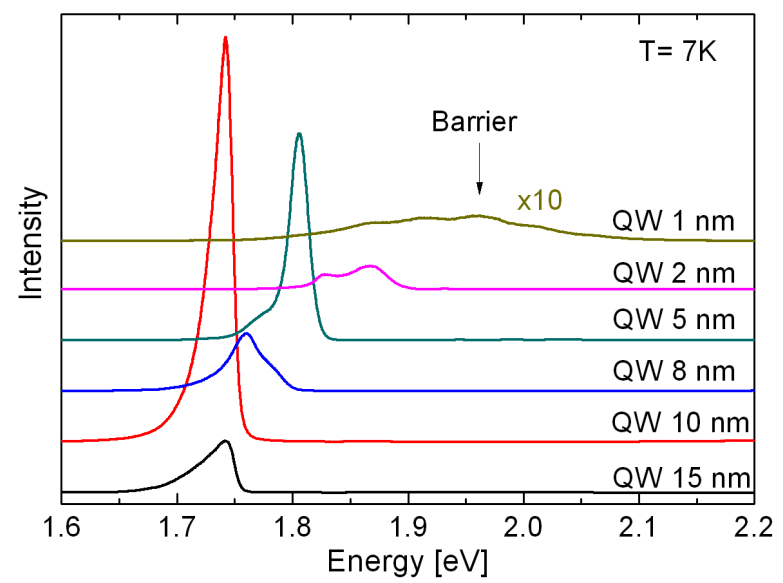

Fig. 3. Helium temperature PL spectra $\mathrm{CdSe} / \mathrm{Cd}_{0.9} \mathrm{Mg}_{0.1} \mathrm{Se}$ QWs with a thickness ranging from 1 to $15 \mathrm{~nm}$. The most intense PL emission is observed for $10 \mathrm{~nm}$ and $5 \mathrm{~nm}$ thick QWs. The latter exhibit the less defect emission.

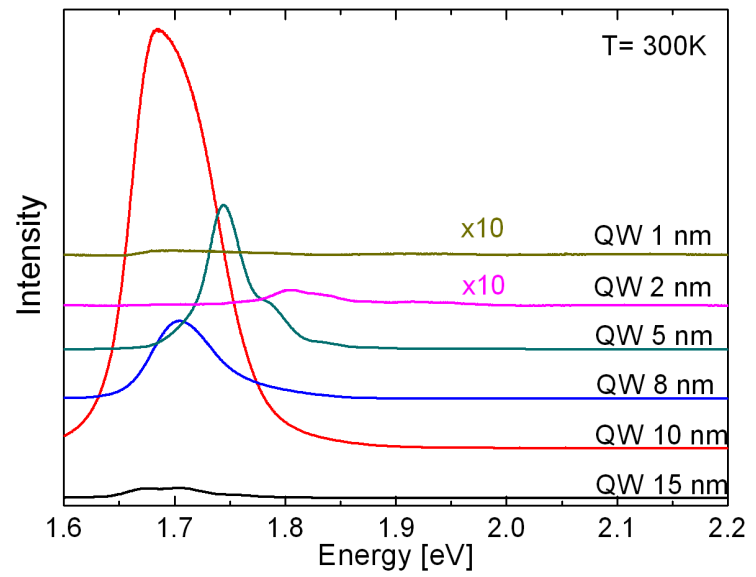

Fig. 4. Room temperature PL spectra $\mathrm{CdSe} / \mathrm{Cd}_{0.9} \mathrm{Mg}_{0.1} \mathrm{Se} \mathrm{QWs}$ with a thickness ranging from 1 to $15 \mathrm{~nm}$. Compared to helium temperature PL measurements, the peaks are widened. The $10 \mathrm{~nm}$ thick QW exhibit the most intense PL emission.

In order to obtain a higher energy of photoluminescence we decided to grow quantum wells containing $10 \%$ magnesium in $15 \%$ barrier and multi-quantum well (MQW) consisting of ten $1 \mathrm{~nm}$ CdSe thick QWs separated by $2 \mathrm{~nm} \mathrm{Cd} \mathrm{Cd}_{0.75} \mathrm{Mg}_{0.25}$ Se layers in order to increase the energy of barrier. At low temperature $(7 \mathrm{~K})$ we observe that the addition of $10 \%$ of magnesium shifts the emission line respectively from 1.8 to $2.01 \mathrm{eV}$ and from 1.74 to $1.95 \mathrm{eV}$ for the $5 \mathrm{~nm}$ and $10 \mathrm{~nm}$ thick QWs. In 
case of the MQW, we observe the emission energy of $2.11 \mathrm{eV}$ at $7 \mathrm{~K}$, which is placed already in the yellow spectral range (above $2.07 \mathrm{eV}$ ) (Fig. 5) and PL intensity is still strong enough at room temperature (Fig. 6) to use this structure as yellow light emitter. We note that yellow range is inaccessible for bright structures with ZnSe barriers, such as CdSe/ZnSe QDs with PL energy above $2.3 \mathrm{eV}$ [23].

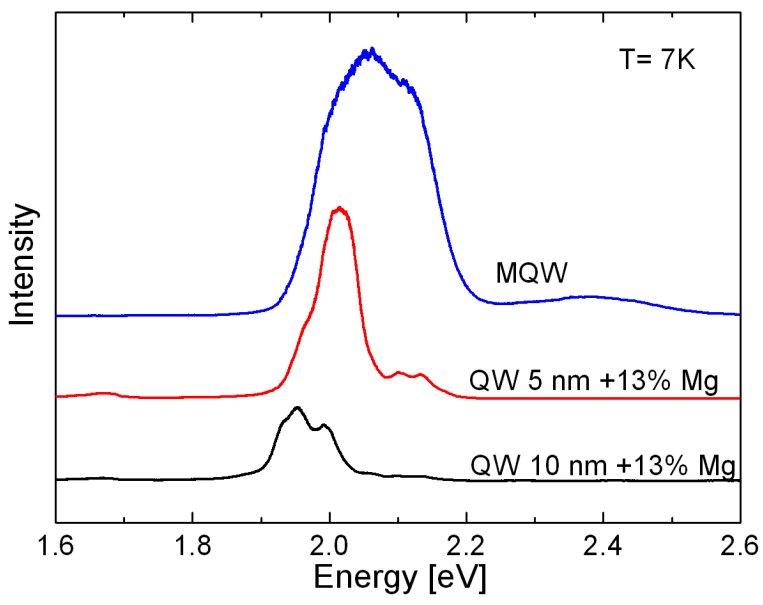

Fig. 5. Helium temperature PL spectra of the 5 and $10 \mathrm{~nm}$ thick $\mathrm{Cd}_{0.9} \mathrm{Mg}_{0.1} \mathrm{Se} / \mathrm{Cd}_{0.85} \mathrm{Mg}_{0.15}$ Se QWs and MQW emitting in the yellow range.

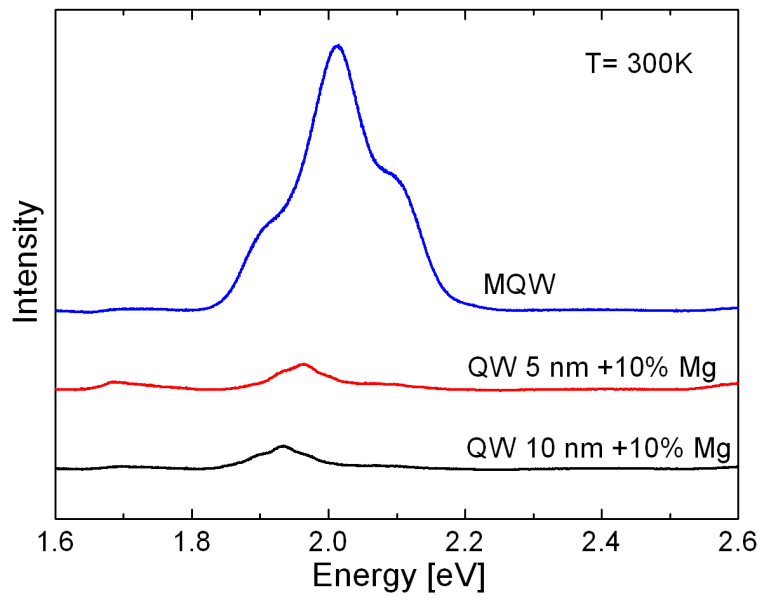

Fig. 6. Room temperature PL spectra of the 5 and $10 \mathrm{~nm}$ thick $\mathrm{Cd}_{0.9} \mathrm{Mg}_{0.1} \mathrm{Se} / \mathrm{Cd}_{0.85} \mathrm{Mg}_{0.15} \mathrm{Se} \mathrm{QWs}$ and MQW. The MQW exhibit a much more robust PL emission up to room temperature.

Temperature dependence of PL intensity is shown in Fig. 7. Integrated PL peaks of $10 \mathrm{~nm}$ QW, $5 \mathrm{~nm}$ QW, and multi-QW are presented, as a function of temperature in the range $10-300 \mathrm{~K}$. It appears that the temperature dependence is similar for all presented structures. This suggests that origin of main PL peaks in all structures is the same and is related to QWs optical transitions.
Slightly weaker variation is observed for structures with deeper binding potential, so for wider QWs.

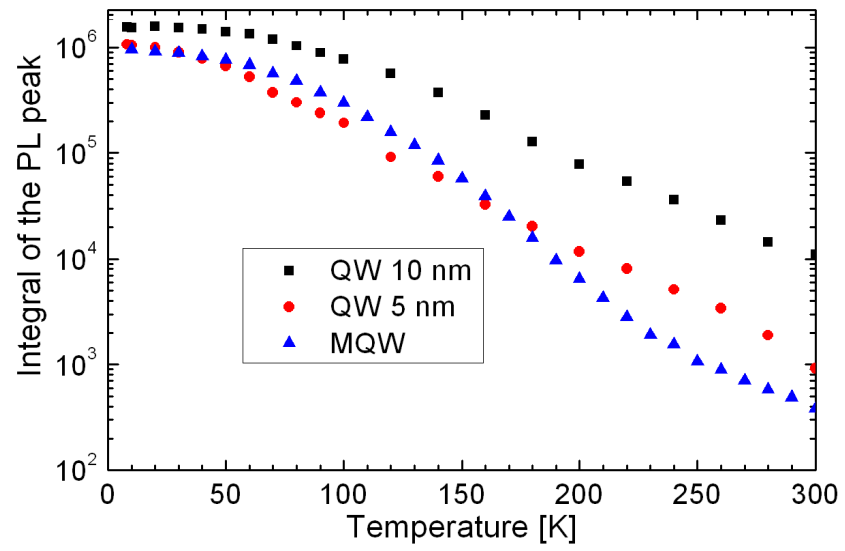

Fig. 7. Intensity of the PL intensity depending on temperature of the 5 and $10 \mathrm{~nm}$ thick $\mathrm{CdSe} / \mathrm{Cd}_{0.9} \mathrm{Mg}_{0.1} \mathrm{Se}$ QWs and MQW.

\section{Conclusions}

We used the band gap engineering methods to design and grow by MBE various CdSe QWs. The optical characterization allowed us to tune up this structure to emit light in the range of 1.74 to $2.1 \mathrm{eV}$ depending on the QW thickness and composition. The most intense PL (at 7 and $300 \mathrm{~K}$ ) was observed for $10 \mathrm{~nm}$ thick $\mathrm{CdSe} / \mathrm{Cd}_{0.9} \mathrm{Mg}_{0.1}$ Se QWs. Such a structure also exhibits a sharp emission line (FWHM $=20 \mathrm{meV})$ at helium temperature.

\section{Acknowledgments}

Acknowledgements This work was supported by the Polish National Science Centre under decisions DEC-2013/09/B/ST3/02603 and DEC2011/02/A/ST3/00131, by the Polish National Centre for Research and Development project LIDER, and by the Foundation for Polish Science (FNP) subsidy "Mistrz". Project was carried out with the use of CePT, CeZaMat, and NLTK infrastructures financed by the European Union - the European Regional Development Fund within the Operational Programme "Innovative economy" for 2007 - 2013.

\section{References}

[1] W. Pacuski, C. Kruse, S. Figge, D. Hommel, Appl. Phys. Lett. 94, 191108 (2009).

[2] C. Kruse, W. Pacuski, T. Jakubczyk, J. Kobak, J.A. Gaj, K. Frank, M. Schowalter, A. Rosenauer, M. Florian, F. Jahnke, D. Hommel, Nanotechnology 22, 285204 (2011).

[3] M. Ściesiek, K. Gietka, A. Golnik, P. Kossacki, T. Jakubczyk, W. Pacuski, C. Kruse, D. Hommel, Acta Phys. Pol. A 120, 877 (2011). 
[4] W. Pacuski, T. Jakubczyk, C. Kruse, J. Kobak, T. Kazimierczuk, M. Goryca, A. Golnik, P. Kossacki, M. Wiater, P. Wojnar, G. Karczewski, T. Wojtowicz, D. Hommel, Cryst. Growth Des. 14, 988 (2014).

[5] T. Jakubczyk, W. Pacuski, T. Smoleński, A. Golnik, M. Florian, F. Jahnke, C. Kruse, D. Hommel, P. Kossacki, Appl. Phys. Lett. 101, 132105 (2012).

[6] T. Jakubczyk, W. Pacuski, T. Smoleński, A. Golnik, M. Florian, F. Jahnke, C. Kruse, D. Hommel, P. Kossacki, J. Appl. Phys. 113, 136504 (2013).

[7] T. Jakubczyk, W. Pacuski, P. Duch, P. Godlewski, A. Golnik, C. Kruse, D. Hommel, J.A. Gaj, Cent. Eur. J. Phys. 9, 428 (2011).

[8] C. Weisbuch, M. Nishioka, A. Ishikawa, Y. Arakawa, Phys. Rev. Lett. 69, 3314 (1992).

[9] R. Houdré, R.P. Stanley, U. Oesterle, M. Ilegems, C. Weisbuch, Phys. Rev. B 49, 16761 (1994).

[10] J.-G. Rousset, J. Kobak, E. Janik, T. Jakubczyk, R. Rudniewski, P. Piotrowski, M. Ściesiek, J. Borysiuk, T. Slupinski, A. Golnik, P. Kossacki, M. Nawrocki, W. Pacuski, J. Cryst. Growth 401, 499 (2014).

[11] S. Boyer-Richard, C. Robert, L. Gérard, J.P. Richters, R. André, J. Bleuse, H. Mariette, J. Evenand, J.M. Jancu, Nanoscale Res. Lett. 7, 543 (2012).

[12] J. Haetty, E.H. Lee, H. Luo, A. Petrou, J. Warnock, Solid State Commun. 108, 205 (1998).

[13] G. Kalpana, B. Palanivel, R.M. Thomas, M. Rajagopalan, Physica B 222, 223 (1996).

[14] R. Pandey, A. Sutjianto, Solid State Commun. 91, 269 (1994).
[15] A. Kuhn, A. Chevy, M.-J. Naud, J. Cryst. Growth 9, 263 (1971).

[16] W. Paszkowicz, J. Szatkowski, Powder Diffr. 13, 50 (1998).

[17] Y.M. Park, R. Andre, J. Kasprzak, Le Si Dang, E. Bellet-Amalric, Appl. Surf. Sci. 253, 6946 (2007).

[18] U. Manna, Q. Zhang, S. Dhomkar, I.F. Salakhutdinov, M.C. Tamargo, I.C. Noyan, G.F. Neumark, I.L. Kuskovsky, J. Appl. Phys. 112, 063521 (2012).

[19] P. Feng, F. Gard, J.D. Riley, P.J. Pigram, R. Leckey, Th. Seyller, L. Ley, J. Electron. Spectrosc. Relat. Phenom. 114-116, 527 (2001).

[20] M. Wörz, E. Griebl, Th. Reisinger, R. Flierl, B. Haserer, T. Semmler, T. Frey, W. Gebhardt, Phys. Status Solidi 202, 805 (1997).

[21] J.-H. Yang, S.Y. Chen, W.-J. Yin, X.G. Gong, A. Walsh, S.-H. Wei, Phys. Rev. B 79, 245202 (2009).

[22] F. Firszt, S. Łęgowski, H. Męczyńska, J. Szatkowski, W. Paszkowicz, M. Marczak, J. Cryst. Growth 184/185, 1053 (1998).

[23] J. Kobak, T. Smoleński, M. Goryca, M. Papaj, K. Gietka, A. Bogucki, M. Koperski, J.-G. Rousset, J. Suffczyński, E. Janik, M. Nawrocki, A. Golnik, P. Kossacki, W. Pacuski, Nat. Commun. 5, 3191 (2014). 